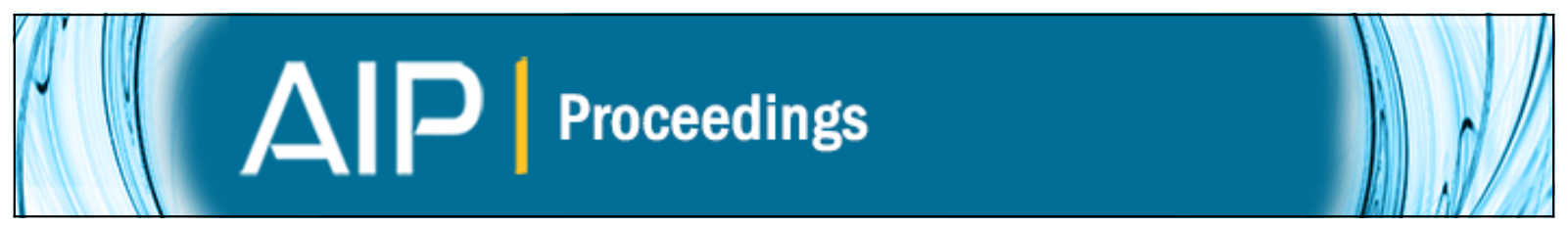

\title{
Recent advances in the numerical solution of Hamiltonian PDEs
}

Luigi Brugnano, Gianluca Frasca Caccia, and Felice lavernaro

Citation: AIP Conference Proceedings 1648, 150008 (2015); doi: 10.1063/1.4912438

View online: http://dx.doi.org/10.1063/1.4912438

View Table of Contents: http://scitation.aip.org/content/aip/proceeding/aipcp/1648?ver=pdfcov

Published by the AIP Publishing

\section{Articles you may be interested in}

Energy conservation issues in the numerical solution of Hamiltonian PDEs

AIP Conf. Proc. 1648, 020002 (2015); 10.1063/1.4912306

Recent advances in the numerical solution of conservative problems

AIP Conf. Proc. 1493, 175 (2012); 10.1063/1.4765487

Convergence in numerical solutions of the Dicke Hamiltonian

AIP Conf. Proc. 1488, 418 (2012); 10.1063/1.4759424

A Cell-Based Finite Difference Method for the Numerical Solution of PDEs

AIP Conf. Proc. 1368, 69 (2011); 10.1063/1.3663462

Recent Advances in Homogeneous Herz Spaces and PDE's

AIP Conf. Proc. 1168, 1492 (2009); 10.1063/1.3241380 


\title{
Recent Advances in the Numerical Solution of Hamiltonian PDEs
}

\author{
Luigi Brugnano*, Gianluca Frasca Caccia* and Felice Iavernaro ${ }^{\dagger}$ \\ *Dipartimento di Matematica e Informatica "U. Dini”, Università di Firenze, Italy \\ ${ }^{\dagger}$ Dipartimento di Matematica, Università di Bari, Italy
}

\begin{abstract}
The numerical solution of Hamiltonian PDEs has been the subject of many investigations in the last years, specially concerning the use of multi-symplectic methods. We shall here be concerned with the use of energy-conserving methods in the HBVMs class, when a spectral space discretization is considered.
\end{abstract}

Keywords: Hamiltonian Partial Differential Equations, Fourier discretization, Energy-conserving Runge-Kutta methods, Hamiltonian Boundary Value Methods.

PACS: 02.60.-x; 45.20.dh; 45.20.Jj; 02.30.Hq; 02.70.Jn; 02.70.Bf. MSC: 65P10; 65L05; 65M20.

\section{INTRODUCTION}

The efficient numerical solution of Hamiltonian PDEs has been the subject of many investigations in recent years (see, e.g., $[15,1]$ ) especially in connection with the use of multi-symplectic methods $[13,14]$. Here, we shall be concerned with the use of energy-conserving methods in the HBVMs class $[5,6,8]$, which are methods based on the concept of discrete line integral [10,11, 12], when they are coupled with a spectral space discretization (see, e.g., [9]). We shall sketch the approach when periodic boundary conditions are prescribed, even though the used approach can be generalized to the case of general boundary conditions. For simplicity, though without loss of generality, we shall consider the 1D nonlinear wave equation,

$$
\begin{aligned}
u_{t t}(x, t) & =u_{x x}(x, t)-f^{\prime}(u(x, t)), \quad(x, t) \in(0,1) \times(0, \infty), \\
u(x, 0) & =\psi_{0}(x), \quad u_{t}(x, 0)=\psi_{1}(x), \quad x \in(0,1), \\
u(0, t) & =u(1, t), \quad t>0 .
\end{aligned}
$$

As usual, subscripts denote partial derivatives, and the functions $f, \psi_{0}$ and $\psi_{1}$ are supposed to be sufficiently smooth, so they define a regular solution $u(x, t)\left(f^{\prime}\right.$ denotes the derivative of $\left.f\right)$. By setting $v=u_{t}$, and defining the functional

$$
\mathscr{H}[u, v](t)=\int_{0}^{1}\left[\frac{1}{2} v^{2}(x, t)+\frac{1}{2} u_{x}^{2}(x, t)+f(u(x, t))\right] \mathrm{d} x \equiv \int_{0}^{1} E(x, t) \mathrm{d} x,
$$

we can rewrite (1) as the infinite-dimensional Hamiltonian system

$$
\begin{aligned}
u_{t}(x, t) & =v(x, t), \quad(x, t) \in(0,1) \times(0, \infty), \\
v_{t}(x, t) & =u_{x x}(x, t)-f^{\prime}(u(x, t)),
\end{aligned}
$$

that is, the first-order formulation of the first equation in (1), for which (2) is a conserved quantity. In view of applying a Fourier spatial discretization for problem (1), let us consider the following orthonormal functions in $[0,1]$ :

$$
c_{0}(x) \equiv 1, \quad c_{k}(x)=\sqrt{2} \cos (2 k \pi x), \quad s_{k}(x)=\sqrt{2} \sin (2 k \pi x), \quad k=1,2, \ldots,
$$

so that

$$
\int_{0}^{1} c_{i}(x) c_{j}(x) \mathrm{d} x=\int_{0}^{1} s_{i}(x) s_{j}(x) \mathrm{d} x=\delta_{i j}, \quad \int_{0}^{1} c_{i}(x) s_{j}(x) \mathrm{d} x=0, \quad \forall i, j,
$$

$\delta_{i j}$ being the Kronecker symbol. Then, the following expansion of the solution of (1) is a slightly different way of writing the usual Fourier expansion in space:

$$
u(x, t)=c_{0}(x) \gamma_{0}(t)+\sum_{n \geq 1}\left[c_{n}(x) \gamma_{n}(t)+s_{n}(x) \eta_{n}(t)\right] \equiv \gamma_{0}(t)+\sum_{n \geq 1}\left[c_{n}(x) \gamma_{n}(t)+s_{n}(x) \eta_{n}(t)\right], \quad x \in[0,1], t \geq 0 .
$$


Consequently, by taking into account (3)-(4), the first equation in (1) can be rewritten as:

$$
\begin{aligned}
& \ddot{\gamma}_{n}(t)=-(2 \pi n)^{2} \gamma_{n}(t)-\int_{0}^{1} c_{n}(x) f^{\prime}\left(\gamma_{0}(t)+\sum_{j \geq 1}\left[c_{j}(x) \gamma_{j}(t)+s_{j}(x) \eta_{j}(t)\right]\right) \mathrm{d} x, \quad n \geq 0, \\
& \ddot{\eta}_{n}(t)=-(2 \pi n)^{2} \eta_{n}(t)-\int_{0}^{1} s_{n}(x) f^{\prime}\left(\gamma_{0}(t)+\sum_{j \geq 1}\left[c_{j}(x) \gamma_{j}(t)+s_{j}(x) \eta_{j}(t)\right]\right) \mathrm{d} x, \quad n \geq 1,
\end{aligned}
$$

where the dot denotes, as usual, the time derivative. By introducing the infinite vectors

$$
\mathbf{q}(t)=\left(\gamma_{0}(t), \gamma_{1}(t), \eta_{1}(t), \gamma_{2}(t), \eta_{2}(t), \ldots\right)^{\top}, \quad \mathbf{p}(t)=\dot{\mathbf{q}}(t), \quad \boldsymbol{\omega}(x)=\left(c_{0}(x), c_{1}(x), s_{1}(x), c_{2}(x), s_{2}(x), \ldots\right)^{\top},
$$

the infinite matrix $D=\operatorname{diag}\left(0,(2 \pi)^{2},(2 \pi)^{2},(4 \pi)^{2},(4 \pi)^{2}, \ldots\right)$, and considering that $u(x, t)=\boldsymbol{\omega}(x)^{\top} \mathbf{q}(t)$, problem (6) can be cast in vector form as the following Hamiltonian problem:

$$
\dot{\mathbf{q}}(t)=\mathbf{p}(t), \quad \dot{\mathbf{p}}(t)=-D \mathbf{q}(t)-\int_{0}^{1} \boldsymbol{\omega}(x) f^{\prime}\left(\boldsymbol{\omega}(x)^{\top} \mathbf{q}(t)\right) \mathrm{d} x, \quad t \geq 0,
$$

with Hamiltonian

$$
H(\mathbf{q}, \mathbf{p})=\frac{1}{2} \mathbf{p}^{\top} \mathbf{p}+\frac{1}{2} \mathbf{q}^{\top} D \mathbf{q}+\int_{0}^{1} f\left(\boldsymbol{\omega}(x)^{\top} \mathbf{q}\right) \mathrm{d} x,
$$

which is easily seen to be equivalent to the Hamiltonian (2), via the expansion (5).

\section{TRUNCATED FOURIER APPROXIMATION}

In the computational practice, it is mandatory to truncate the infinite expansion (5) to a finite sum:

$$
u(x, t) \approx \gamma_{0}(t)+\sum_{n=1}^{N}\left[c_{n}(x) \gamma_{n}(t)+s_{n}(x) \eta_{n}(t)\right] \equiv u_{N}(x, t) .
$$

This reflects in the fact that the differential equations (6) now reduce to a finite number, i.e., $2 N+1$. Correspondingly, one defines the vectors $\mathbf{q}_{N}(t), \mathbf{p}_{N}(t), \boldsymbol{\omega}_{N}(t) \in \mathbb{R}^{2 N+1}$ given by the leading parts of the corresponding infinite vectors in (7), and the principal submatrix $D_{N}$ of dimension $2 N+1$ of matrix $D$. Then, considering that $u_{N}(x, t)=$ $\boldsymbol{\omega}_{N}(x)^{\top} \mathbf{q}_{N}(t)$, the equations which must be satisfied by (9) can be cast in vector form as:

$$
\dot{\mathbf{q}}_{N}(t)=\mathbf{p}_{N}(t), \quad \dot{\mathbf{p}}_{N}(t)=-D_{N} \mathbf{q}_{N}(t)-\int_{0}^{1} \boldsymbol{\omega}_{N}(x) f^{\prime}\left(\boldsymbol{\omega}_{N}(x)^{\top} \mathbf{q}_{N}(t)\right) \mathrm{d} x, \quad t \geq 0,
$$

which is again in Hamiltonian form, with Hamiltonian

$$
H\left(\mathbf{q}_{N}, \mathbf{p}_{N}\right)=\frac{1}{2} \mathbf{p}_{N}^{\top} \mathbf{p}_{N}+\frac{1}{2} \mathbf{q}_{N}^{\top} D_{N} \mathbf{q}_{N}+\int_{0}^{1} f\left(\boldsymbol{\omega}_{N}(x)^{\top} \mathbf{q}_{N}\right) \mathrm{d} x .
$$

This latter is glaringly equivalent to a truncated Fourier expansion of the Hamiltonian (2), that is, by truncating the expansion (5) as done in (9). For approximating problem (10), one can consider the use of a $\operatorname{HBVM}(k, s)$ method, which is the $k$-stage Runge-Kutta method defined by the following Butcher tableau $[6,8]$ :

$$
\begin{array}{c|c}
c_{1} & {\left[b_{j} \sum_{\ell=0}^{s-1} P_{\ell}\left(c_{j}\right) \int_{0}^{c_{i}} P_{j}(\tau) \mathrm{d} \tau\right]_{i, j=1, \ldots, k},}
\end{array} \quad \int_{0}^{1} P_{i}(x) P_{j}(x) \mathrm{d} x=\delta_{i j}, \quad P_{i} \in \Pi_{i}, \quad i, j=0, \ldots, k,
$$

with $P_{i}, P_{j}$ the normalized and shifted Legendre polynomials, and $\left(b_{i}, c_{i}\right)_{i=1, \ldots, k}$ the Legendre weights and abscissae. For such methods, which reduce to the $s$-stage Gauss-Legendre collocation methods when $k=s$, the following result holds true [4].

Theorem 1 For all $k \geq s$, a HBVM(k,s) method applied to problem (10)-(11):

- has order $2 s$; 

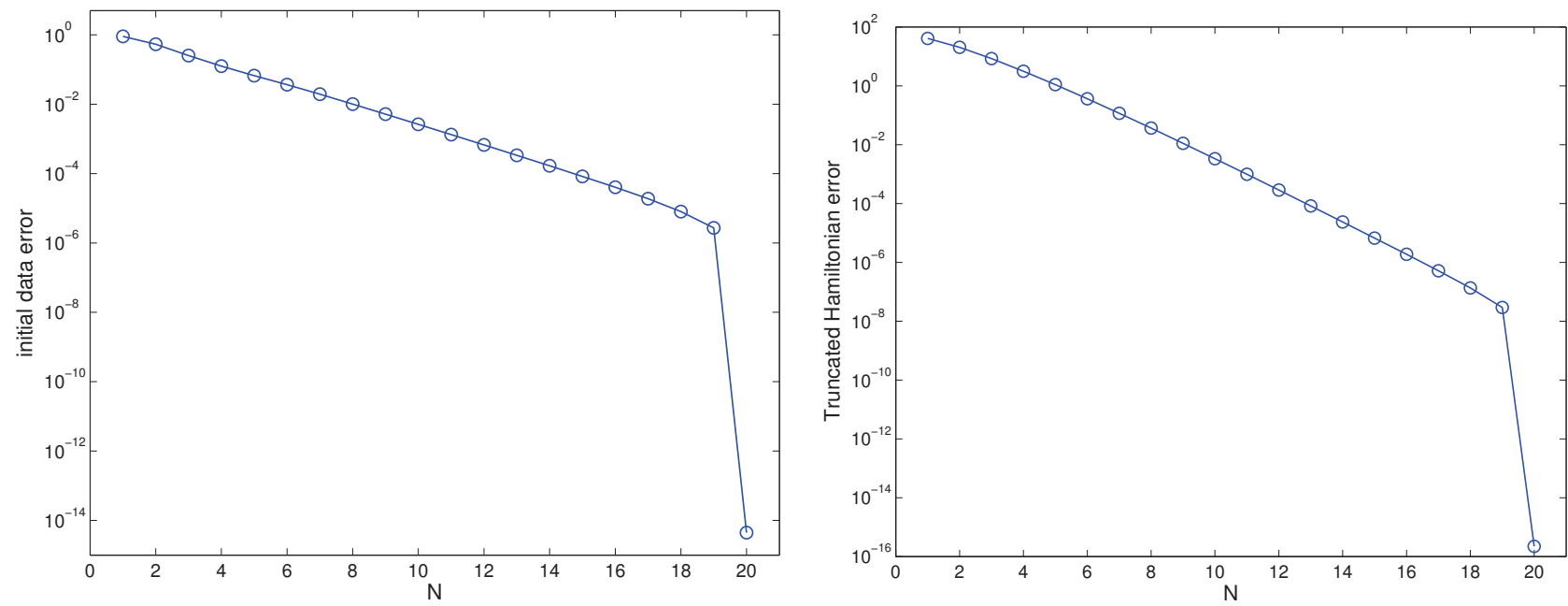

\section{FIGURE 1.}

- is energy conserving when $f$ is a polynomial of degree $v \leq\lfloor 2 k / s\rfloor$;

- for general and suitably regular functions $f$, the energy error at each step is $O\left(h^{2 k+1}\right)$.

This, in turn, implies that a (at least practical) conservation of energy is obtained, provided that $f$ is suitably regular (which will be assumed hereafter), by choosing $k$ large enough, also considering that the computational cost for implementing a $\operatorname{HBVM}(k, s)$ method depends essentially on $s$, rather than on $k[7,2,3]$. Clearly, the integrals appearing at the right-hand side in (10) and (11) can be computed accurately, by using a composite trapezoidal rule, the argument being a periodic function: fo sake of brevity, we shall omit here the handling of such a standard stuff.

We end this section, by stressing that the above arguments can be extended to cope with different kind of boundary conditions, by suitably modifying the Hamiltonian function defining the problem [4].

\section{NUMERICAL TESTS}

The use of energy-conserving methods is quite well understood, proving to be very useful, when speaking about Hamiltonian ordinary differential equations. Their use in the framework of Hamiltonian partial differential equations is fairly less obvious and deserves further investigations. We here just report a few numerical tests, showing that the proposed truncated Fourier space discretization, coupled with a HBVM solution in time, allows to obtain energyconservation, as above described. We consider problem (1) with:

$$
f(u)=u^{4}-u, \quad \psi_{0}(x)=\sum_{n=1}^{20} 2^{-n}[\sin (2 n \pi x)-\cos (2 n \pi x)], \quad \psi_{1}(x) \equiv 0,
$$

for which the Hamiltonian $(2)$ has a value $H_{0} \approx 62.93$. In Figure 1, left-plot, we show the error in the initial data, depending on the value $N$ for the truncation in (9). The right-plot in the same figure displays the error of the corresponding truncated Hamiltonian (11), with respect to the exact Hamltonian (8): as one may infer from the initial data in (12), the error at $N=20$ has to be of the order of round-off errors in both cases.

Let us now solve the same problem, by using a sixth-order $\operatorname{HBVM}(k, 3)$ method, over the interval $[0,100]$. For this purpose, we fix a stepsize $h=10^{-1}$. In the left-plot of Figure 2 we plot the absolute error in the numerical Hamiltonian $\left(\left|H-H_{0}\right|\right)$ when $k=3,5,7:{ }^{1}$ as one may see, the larger $k$ the smaller the error, which is within round-off for $k=7$. In the right-plot in the same figure, one finds the maximum error in the numerical Hamiltonian (line with circles),

$$
E_{H}(k)=\max _{n=1, \ldots, 1000}\left|H_{n}-H_{0}\right|, \quad k=3, \ldots, 9,
$$

\footnotetext{
${ }^{1}$ By the way, when $k=3, \operatorname{HBVM}(3,3)$ coincides with the symplectic 3-stage Gauss-Legendre collocation method
} 

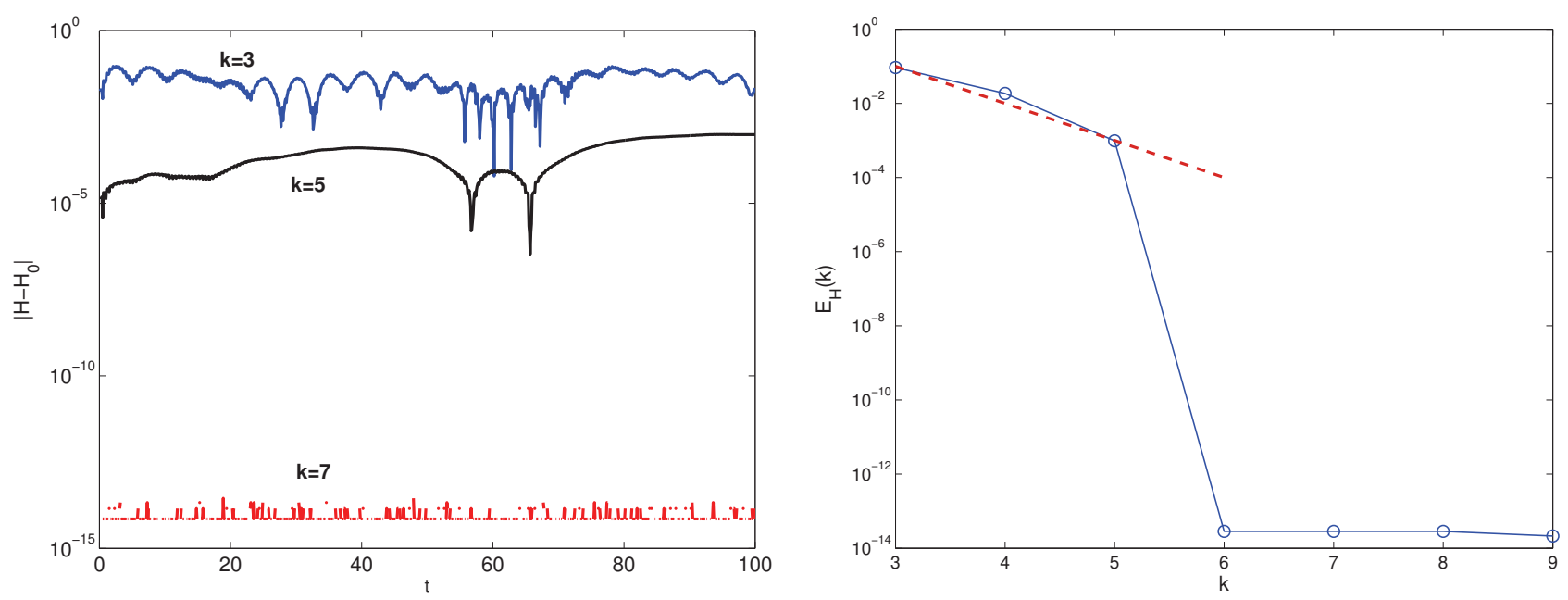

FIGURE 2.

where $H_{n}$ is the value of the numerical Hamiltonian at the time-step $n$. In the same sub-plot, one finds a dashed-line, showing a decrease proportional to $h^{2 k}\left(h=10^{-1}\right.$, in our case). It is then clear that the $E_{H}(k)$ behaves as $h^{2 k}$, for $k=3,4,5$, whereas it is of the order of round-off errors, for $k \geq 6$, according to Theorem 1 . Indeed, in the present case the function $f$ in (12) is a polynomial of degree 4 , and $2 k \geq 4 s \equiv 12$, for $k \geq 6$.

\section{REFERENCES}

1. T.J. Bridges, S. Reich. Numerical methods for Hamiltonian PDEs. J. Phys. A: Math. Gen. 39 (2006) 5287-5320.

2. L. Brugnano, G. Frasca Caccia, F. Iavernaro. Efficient implementation of geometric integrators for separable Hamiltonian problems. AIP Conf. Proc. 1588 (2013) 734-737.

3. L. Brugnano, G. Frasca Caccia, F. Iavernaro. Efficient implementation of Gauss collocation and Hamiltonian Boundary Value Methods. Numer. Algor. 65 (2014) 633-650.

4. L. Brugnano, G. Frasca Caccia, F. Iavernaro. Energy conserving methods, based on spectral space decomposition, for Hamiltonian PDEs. (in progress).

5. L. Brugnano, F. Iavernaro, D. Trigiante. Hamiltonian BVMs (HBVMs): a family of "drift-free" methods for integrating polynomial Hamiltonian systems. AIP Conf. Proc. 1168 (2009) 715-718.

6. L. Brugnano, F. Iavernaro, D. Trigiante. Hamiltonian Boundary Value Methods (Energy Preserving Discrete Line Integral Methods). J. Numer. Anal. Ind. Appl. Math. 5,1-2 (2010) 17-37.

7. L. Brugnano, F. Iavernaro, D. Trigiante. A note on the efficient implementation of Hamiltonian BVMs. J. Comput. Appl. Math. 236 (2011) 375-383.

8. L. Brugnano, F. Iavernaro, D. Trigiante. A simple framework for the derivation and analysis of effective one-step methods for ODEs. Appl. Math. Comput. 218 (2012) 8475-8485.

9. C. Canuto, M.Y. Hussaini, A. Quarteroni, T.A.Zang. Spectral Methods - Evolution to Complex Geometries and Applications to Fluid Dynamics. Springer, 2007.

10. F. Iavernaro, B. Pace. $s$-Stage trapezoidal methods for the conservation of Hamiltonian functions of polynomial type. AIP Conf. Proc. 936 (2007) 603-606.

11. F. Iavernaro, B. Pace. Conservative Block Boundary Value Methods for the Solution of Polynomial Hamiltonian Systems. AIP Conf. Proc. 1048 (2007) 888-891.

12. F. Iavernaro, D. Trigiante. High-order symmetric schemes for the energy conservation of polynomial Hamiltonian problems, J. Numer. Anal. Ind. Appl. Math. 4 (1-2) (2009) 87-101.

13. A.L. Islas, C.M. Schober. Backward error analysis for multisymplectic discretizations of Hamiltonian PDEs. Mathematic and Computers in Simulation 69 (2005) 290-303.

14. A.L. Islas, C.M. Schober. Conservation properties of multisymplectic integrators. Future Generation Computer Systems 22 (2006) 412-422.

15. B. Leimkulher, S. Reich. Simulating Hamiltonian Dynamics. Cambridge University Press, 2004. 